\title{
Continuous Functions with Complicated Local Structure Defined in Terms of Alternating Cantor Series Representation of Numbers
}

\author{
S.O. Serbenyuk \\ Institute of Mathematics of the National Academy of Sciences of Ukraine \\ 3 Tereschenkivska Str., Kyiv-4 01004, Ukraine \\ E-mail: simon.mathscience@imath.kiev.ua, simon6@ukr.net
}

Received October 22, 2015, revised May 18, 2016

\begin{abstract}
The paper is devoted to one infinite parametric class of continuous functions with complicated local structure such that these functions are defined in terms of alternating Cantor series representation of numbers. The main attention is given to differential, integral and other properties of these functions. Conditions of monotony and nonmonotony are found. The functional equations system such that the function from the given class of functions is a solution of the system is indicated.
\end{abstract}

Key words: alternating Cantor series, functional equations system, monotonic function, continuous nowhere monotonic function, singular function, nowhere differentiable function, distribution function.

Mathematics Subject Classification 2010: 39B72, 26A27, 26A30, 11B34, $11 \mathrm{~K} 55$.

\section{Introduction}

For modeling the functions with complicated local structure (singular, continuous nowhere differentiable, nowhere monotonic functions), various representations of real numbers are used widely in modern scientific researches $[1,2,4,9]$. For example, the representations of numbers by positive and alternating series, whose terms are reciprocal to positive integers or their products.

In the present paper, the application of the expansions of real numbers in infinite series, whose terms are rational, to the construction of monotonic singular and continuous nowhere monotonic functions is considered. Traditionally, the simplest examples of these series are $s$-adic and nega-s-adic expansions. 
In 1869, the first time Georg Cantor [10] considered the expansions of real numbers from $[0 ; 1]$ in the positive series

$$
\sum_{n=1}^{\infty} \frac{\varepsilon_{n}}{d_{1} d_{2} \ldots d_{n}}
$$

where $\left(d_{n}\right)$ is a fixed sequence of positive integers $d_{n}>1$ and $\left(A_{d_{n}}\right)$ is a sequence of alphabets $A_{d_{n}} \equiv\left\{0,1, \ldots, d_{n}-1\right\}, \varepsilon_{n} \in A_{d_{n}}$.

In scientific literature the last-mentioned series is called the positive Cantor series or Cantor series. It is easy to see that the representation of real numbers by the Cantor series is a generalization of the $s$-adic numeral system. So it would be logical to assume the possibility of representation of real numbers by the alternating Cantor series

$$
\sum_{n=1}^{\infty} \frac{(-1)^{n} \varepsilon_{n}}{d_{1} d_{2} \ldots d_{n}}
$$

that $[6]$ is a generalization of the nega-s-adic representation of real numbers.

Since 1869, the problem on necessary and sufficient conditions of the rationality of numbers defined by the positive Cantor series has remained open. The last-mentioned problem was solved by the author of the present paper in [5]. Among the papers on finding the necessary or sufficient conditions of rationality of numbers, represented by the positive Cantor series, we have [11]. In the paper, A. Oppenheim studied mostly sufficient conditions of irrationality of numbers defined not only by the positive series (1), but also (1) with positive and negative terms such that for the last-mentioned series the conditions $\left|\varepsilon_{i}\right|<d_{i}-1$ for $i=1,2,3, \ldots$, and $\varepsilon_{m} \varepsilon_{n}<0$ for some $m>i$ and $n>i$, where $i$ is any fixed integer, are true.

Investigations of the present paper are the generalization of studies of M. V. Pratsiovytyi and A.V. Kalashnikov [3]. The similar functions, whose argument was defined by the positive Cantor series, were studied by the author of the present paper in $[7,8]$.

In the present paper, the main attention is given to the study of the main properties of functions with complicated local structure whose arguments are represented by the alternating Cantor series

$$
\sum_{n=1}^{\infty} \frac{1+\varepsilon_{n}}{d_{1} d_{2} \ldots d_{n}}(-1)^{n+1}, \quad \varepsilon_{n} \in A_{d_{n}},
$$

because the domain of definition of these functions is a closed interval $[0 ; 1]$ and each number from the interval can be represented by the last-mentioned series. 
Before turning to the basic research results, let us consider the problem on belonging of expansions (2) and (3) to numeral systems.

Theorem 1. Each number $x \in\left[a_{0}-1 ; a_{0}\right]$, where

$$
a_{0}=\sum_{n=1}^{\infty} \frac{(-1)^{n+1}}{d_{1} d_{2} \ldots d_{n}}
$$

can be represented by the series (2) in not more than two ways.

Theorem 2. For any $x \in[0 ; 1]$, there exist not more than two different sequences $\left(\varepsilon_{n}\right)$ such that

$$
x=\sum_{n=1}^{\infty} \frac{1+\varepsilon_{n}}{d_{1} d_{2} \ldots d_{n}}(-1)^{n+1} .
$$

The proofs of Theorem 1 and Theorem 2 are similar, because the sequence $\left(d_{n}\right)$ is fixed and it follows that $a_{0}=$ const.

Definition 1.1. The defining of an arbitrary number $x$ from $\left[a_{0}-1 ; a_{0}\right]$ (or from $[0 ; 1])$ by the expansion in the alternating Cantor series (2) (or (3)) is called a nega-D-expansion, where $D \equiv\left(d_{n}\right)$, (or nega- $\left(d_{n}\right)$-expansion) of the number $x$ and is denoted by $x=\Delta_{\varepsilon_{1} \varepsilon_{2} \ldots \varepsilon_{n} \ldots}^{-D}$ (or $x=\Delta_{\varepsilon_{1} \varepsilon_{2} \ldots \varepsilon_{n} \ldots}^{-\left(d_{n}\right)}$ ). The last-mentioned notation is called the nega-D-representation (or nega- $\left(d_{n}\right)$-representation) of the number $x$.

Theorem 1 follows from the next two lemmas.

Lemma 1. For any $x \in\left[a_{0}-1 ; a_{0}\right]$, there exists a sequence $\left(\varepsilon_{n}\right)$ such that the number $x$ can be represented by the series (2).

$\mathrm{P}$ r o o f. It is obvious that

$$
\begin{gathered}
a_{0}=\max \left\{\sum_{n=1}^{\infty} \frac{(-1)^{n} \varepsilon_{n}}{d_{1} d_{2} \ldots d_{n}}\right\} \equiv \Delta_{0\left[d_{2}-1\right] 0\left[d_{4}-1\right] 0\left[d_{6}-1\right] 0 \ldots}^{-D} \\
a_{0}-1=\min \left\{\sum_{n=1}^{\infty} \frac{(-1)^{n} \varepsilon_{n}}{d_{1} d_{2} \ldots d_{n}}\right\} \equiv \Delta_{\left[d_{1}-1\right] 0\left[d_{3}-1\right] 0\left[d_{5}-1\right] 0 \ldots}^{-D}
\end{gathered}
$$

Let $x$ be an arbitrary number from $\left(a_{0}-1 ; a_{0}\right)$,

$$
-\frac{\varepsilon_{1}}{d_{1}}-\sum_{k=2}^{\infty} \frac{d_{2 k-1}-1}{d_{1} d_{2} \ldots d_{2 k-1}}<x \leq-\frac{\varepsilon_{1}}{d_{1}}+\sum_{k=1}^{\infty} \frac{d_{2 k}-1}{d_{1} d_{2} \ldots d_{2 k}}
$$


with $0 \leq \varepsilon_{1} \leq d_{1}-1$, and since

$$
\left[a_{0}-1 ; a_{0}\right]=I_{0}=\bigcup_{i=0}^{d_{1}-1}\left[-\frac{i}{d_{1}}-\sum_{k=2}^{\infty} \frac{d_{2 k-1}-1}{d_{1} d_{2} \ldots d_{2 k-1}} ;-\frac{i}{d_{1}}+\sum_{k=1}^{\infty} \frac{d_{2 k}-1}{d_{1} d_{2} \ldots d_{2 k}}\right],
$$

it is obtained that

$$
-\sum_{k=2}^{\infty} \frac{d_{2 k-1}-1}{d_{1} d_{2} \ldots d_{2 k-1}}<x+\frac{\varepsilon_{1}}{d_{1}} \leq \sum_{k=1}^{\infty} \frac{d_{2 k}-1}{d_{1} d_{2} \ldots d_{2 k}} .
$$

Let $x+\frac{\varepsilon_{1}}{d_{1}}=x_{1}$. Then the following cases are obtained:

1. If the equality

$$
x_{1}=\sum_{k=1}^{\infty} \frac{d_{2 k}-1}{d_{1} d_{2} \ldots d_{2 k}}
$$

holds, then

$$
x=\Delta_{\varepsilon_{1}\left[d_{2}-1\right] 0\left[d_{4}-1\right] 0 \ldots}^{-D} \text { or } x=\Delta_{\left[\varepsilon_{1}-1\right] 0\left[d_{3}-1\right] 0\left[d_{5}-1\right] 0 \ldots}^{-D}
$$

2. If the equality is not true, then $x=-\frac{\varepsilon_{1}}{d_{1}}+x_{1}$, where

$$
\frac{\varepsilon_{2}}{d_{1} d_{2}}-\sum_{k=2}^{\infty} \frac{d_{2 k-1}-1}{d_{1} d_{2} \ldots d_{2 k-1}} \leq x_{1}<\frac{\varepsilon_{2}}{d_{1} d_{2}}+\sum_{k=2}^{\infty} \frac{d_{2 k}-1}{d_{1} d_{2} \ldots d_{2 k}}
$$

In the same way, let $x_{2}=x_{1}-\frac{\varepsilon_{2}}{d_{1} d_{2}}$. Then we have:

1. If the equality

$$
x_{2}=\sum_{k=2}^{\infty} \frac{d_{2 k-1}-1}{d_{1} d_{2} \ldots d_{2 k-1}}
$$

holds, then

$$
x=\Delta_{\varepsilon_{1} \varepsilon_{2}\left[d_{3}-1\right] 0\left[d_{5}-1\right] 0 \ldots}^{-D} \text { or } x=\Delta_{\varepsilon_{1}\left[\varepsilon_{2}-1\right] 0\left[d_{4}-1\right] 0\left[d_{6}-1\right] 0 \ldots}^{-D} .
$$

2. In another case,

$$
\begin{gathered}
x=-\frac{\varepsilon_{1}}{d_{1}}+\frac{\varepsilon_{2}}{d_{1} d_{2}}+x_{2}, \text { where } \\
-\frac{\varepsilon_{3}}{d_{1} d_{2} d_{3}}-\sum_{k=3}^{\infty} \frac{d_{2 k-1}-1}{d_{1} d_{2} \ldots d_{2 k-1}}<x_{2} \leq-\frac{\varepsilon_{3}}{d_{1} d_{2} d_{3}}+\sum_{k=2}^{\infty} \frac{d_{2 k}-1}{d_{1} d_{2} \ldots d_{2 k}}, \text { etc. }
\end{gathered}
$$


So, for the positive integer $m$,

$$
-\sum_{k>\frac{m+2}{2}} \frac{d_{2 k-1}-1}{d_{1} d_{2} \ldots d_{2 k-1}}<x_{m}-\frac{(-1)^{m+1} \varepsilon_{m+1}}{d_{1} d_{2} \ldots d_{m+1}}<\sum_{k>\frac{m+1}{2}} \frac{d_{2 k}-1}{d_{1} d_{2} \ldots d_{2 k}} .
$$

Moreover, the following cases are possible:

1.

$$
x_{m+1}= \begin{cases}\sum_{k>\frac{m+2}{2}} \frac{d_{2 k-1}-1}{d_{1} d_{2} \ldots d_{2 k-1}}, & \text { if } m \text { is an odd number } \\ \sum_{k>\frac{m+1}{2}} \frac{d_{2 k}-1}{d_{1} d_{2} \ldots d_{2 k}}, & \text { if } m \text { is an even number. }\end{cases}
$$

In this case,

$$
x=\Delta_{\varepsilon_{1} \varepsilon_{2} \ldots \varepsilon_{m+1}}^{-D}\left[d_{m+2}-1\right] 0\left[d_{m+4}-1\right] 0 \ldots
$$

or

$$
x=\Delta_{\varepsilon_{1} \ldots \varepsilon_{m}\left[\varepsilon_{m+1}-1\right] 0\left[d_{m+3}-1\right] 0\left[d_{m+5}-1\right] 0 \ldots}^{-D} .
$$

2. If there does not exist $m \in \mathbb{N}$ such that the last-mentioned system is true, then

$$
x=\sum_{n=1}^{m+1} \frac{(-1)^{n} \varepsilon_{n}}{d_{1} d_{2} \ldots d_{n}}+x_{m+1} .
$$

Continuing the process indefinitely, we obtain

$$
x=-\frac{\varepsilon_{1}}{d_{1}}+x_{1}=\ldots=-\frac{\varepsilon_{1}}{d_{1}}+\frac{\varepsilon_{2}}{d_{1} d_{2}}-\frac{\varepsilon_{3}}{d_{1} d_{2} d_{3}}+\ldots+\frac{(-1)^{n} \varepsilon_{n}}{d_{1} d_{2} \ldots d_{n}}+x_{n}=\ldots
$$

Hence,

$$
x=\sum_{n=1}^{\infty} \frac{(-1)^{n} \varepsilon_{n}}{d_{1} d_{2} \ldots d_{n}}
$$

Lemma 2. The numbers

$$
x=\Delta_{\varepsilon_{1} \varepsilon_{2} \ldots \varepsilon_{m-1} \varepsilon_{m} \varepsilon_{m+1} \ldots}^{-D} \text { and } x^{\prime}=\Delta_{\varepsilon_{1} \varepsilon_{2} \ldots \varepsilon_{m-1} \varepsilon_{m}^{\prime} \varepsilon_{m+1}^{\prime} \ldots}^{-D},
$$

where $\varepsilon_{m} \neq \varepsilon_{m}^{\prime}$, are equal iff one of the systems

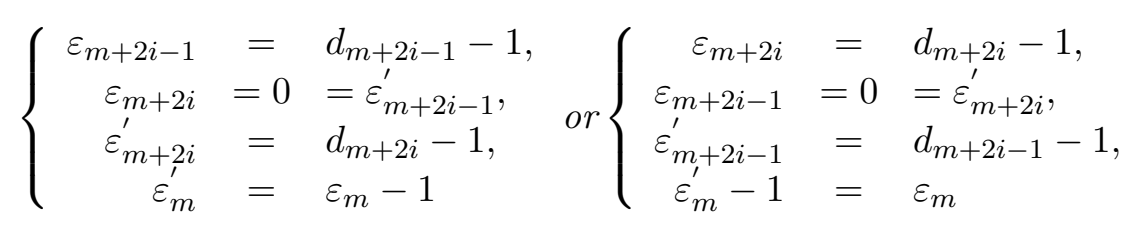

is satisfied for all $i \in \mathbb{N}$. 
P r o o f. Necessity. Let $\varepsilon_{m}=\varepsilon_{m}^{\prime}+1$. Then

$$
\begin{gathered}
0=x-x^{\prime}=\Delta_{\varepsilon_{1} \varepsilon_{2} \ldots \varepsilon_{m-1} \varepsilon_{m} \varepsilon_{m+1} \ldots}^{-D}-\Delta_{\varepsilon_{1} \varepsilon_{2} \ldots \varepsilon_{m-1} \varepsilon_{m}^{\prime} \varepsilon_{m+1}^{\prime} \ldots}^{-D}=\frac{(-1)^{m}}{d_{1} d_{2} \ldots d_{m}} \\
+\frac{(-1)^{m+1}\left(\varepsilon_{m+1}-\varepsilon_{m+1}^{\prime}\right)}{d_{1} d_{2} \ldots d_{m+1}}+\ldots+\frac{\varepsilon_{m+i}-\varepsilon_{m+i}^{\prime}}{d_{1} d_{2} \ldots d_{m+i}}(-1)^{m+i}+\ldots \\
=\frac{(-1)^{m}}{d_{1} d_{2} \ldots d_{m}}\left(1+\sum_{i=1}^{\infty} \frac{(-1)^{i}\left(\varepsilon_{m+i}-\varepsilon_{m+i}^{\prime}\right)}{d_{m+1} d_{m+2} \ldots d_{m+i}}\right) . \\
v \equiv \sum_{i=1}^{\infty} \frac{(-1)^{i}\left(\varepsilon_{m+i}-\varepsilon_{m+i}^{\prime}\right)}{d_{m+1} d_{m+2} \ldots d_{m+i}} \geq-\sum_{i=1}^{\infty} \frac{d_{m+i}-1}{d_{m+1} d_{m+2} \ldots d_{m+i}}=-1 .
\end{gathered}
$$

The last inequality becomes an equality only when

$$
\varepsilon_{m+2 i}=\varepsilon_{m+2 i-1}^{\prime}=0 \text { and } \varepsilon_{m+2 i-1}=d_{m+2 i-1}-1, \varepsilon_{m+2 i}^{\prime}=d_{m+2 i}-1 .
$$

That is, the conditions for the first system follow from the equality $x=x^{\prime}$. It is easy to see that the conditions for the second system follow from $x=x^{\prime}$ under the assumption that $\varepsilon_{m}^{\prime}=\varepsilon_{m}+1$.

It is obvious that the sufficiency is true.

Definition 1.2. A number $x \in[0 ; 1]$ is called a nega- $\left(d_{n}\right)$-rational number if it can be represented by

$\Delta_{\varepsilon_{1} \varepsilon_{2} \ldots \varepsilon_{n-1} \varepsilon_{n}\left[d_{n+1}-1\right] 0\left[d_{n+3}-1\right] 0\left[d_{n+5}-1\right] \ldots}^{-\left(d_{n}\right)}=\Delta_{\varepsilon_{1} \varepsilon_{2} \ldots \varepsilon_{n-1}\left[\varepsilon_{n}-1\right] 0\left[d_{n+2}-1\right] 0\left[d_{n+4}-1\right] 0\left[d_{n+6}-1 \ldots\right.}^{-\left(d_{n}\right)}$.

The rest of the numbers from $[0 ; 1]$ are called nega- $\left(d_{n}\right)$-irrational numbers and have a unique nega- $\left(d_{n}\right)$-representation.

\section{The Object of Research}

Let $P=\left\|p_{i, n}\right\|$ be a given matrix such that $n=1,2, \ldots$ and $i=\overline{0, d_{n}-1}$. For the matrix, the following system of the properties holds:

$$
\left\{\begin{array}{lr}
1^{\circ} . & \forall n \in \mathbb{N}: p_{i, n} \in(-1 ; 1) ; \\
2^{\circ} . & \forall n \in \mathbb{N}: \sum_{i=0}^{d_{n}-1} p_{i, n}=1 ; \\
3^{\circ} . & \forall\left(i_{n}\right), i_{n} \in A_{d_{n}}: \prod_{n=1}^{\infty}\left|p_{i_{n}, n}\right|=0 ; \\
4^{\circ} . & \forall i_{n} \in A_{d_{n}} \backslash\{0\}: 1>\beta_{i_{n}, n}=\sum_{i=0}^{i_{n}-1} p_{i, n}>\beta_{0, n}=0 .
\end{array}\right.
$$


Let $x=\Delta_{\varepsilon_{1} \varepsilon_{2} \ldots \varepsilon_{n} \ldots}^{-\left(d_{n}\right)}$. Consider the function

$$
\tilde{F}(x)=\beta_{\varepsilon_{1}(x), 1}+\sum_{n=2}^{\infty}\left(\tilde{\beta}_{\varepsilon_{n}(x), n} \prod_{j=1}^{n-1} \tilde{p}_{\varepsilon_{j}(x), j}\right),
$$

where

$$
\begin{aligned}
& \tilde{\beta}_{\varepsilon_{n}(x), n}= \begin{cases}\beta_{\varepsilon_{n}(x), n}, & \text { if } n \text { is an odd number, } \\
\beta_{d_{n}-1-\varepsilon_{n}(x), n}, & \text { if } n \text { is an even number, }\end{cases} \\
& \tilde{p}_{\varepsilon_{n}(x), n}= \begin{cases}p_{\varepsilon_{n}(x), n}, & \text { if } n \text { is an odd number } \\
p_{d_{n}-1-\varepsilon_{n}(x), n}, & \text { if } n \text { is an even number. }\end{cases}
\end{aligned}
$$

To study other methods of defining the considered functions, we use the relation between representations of real numbers by the positive Cantor series

$$
\frac{\varepsilon_{1}}{d_{1}}+\frac{\varepsilon_{2}}{d_{1} d_{2}}+\ldots+\frac{\varepsilon_{n}}{d_{1} d_{2} \ldots d_{n}}+\ldots \equiv \Delta_{\varepsilon_{1} \varepsilon_{2} \ldots \varepsilon_{n} \ldots}^{D}
$$

and the alternating Cantor series

$$
\begin{aligned}
\sum_{n=1}^{\infty} \frac{1+\varepsilon_{n}}{d_{1} d_{2} \ldots d_{n}}(-1)^{n+1} & \equiv \Delta_{\varepsilon_{1} \varepsilon_{2} \ldots \varepsilon_{n} \ldots}^{-\left(d_{n}\right)} \equiv \Delta_{\varepsilon_{1}\left[d_{2}-1-\varepsilon_{2}\right] \varepsilon_{3}\left[d_{4}-1-\varepsilon_{4}\right] \ldots}^{D} \\
& \equiv \frac{\varepsilon_{1}}{d_{1}}+\frac{d_{2}-1-\varepsilon_{2}}{d_{1} d_{2}}+\ldots
\end{aligned}
$$

It follows that

$$
\tilde{F}(x)=F(g(x))=F \circ g,
$$

where

$$
\begin{gathered}
x=\Delta_{\varepsilon_{1} \varepsilon_{2} \ldots \varepsilon_{n} \ldots}^{-\left(d_{n}\right)} \stackrel{g}{\rightarrow} \Delta_{\varepsilon_{1}\left[d_{2}-1-\varepsilon_{2}\right] \ldots \varepsilon_{2 n-1}\left[d_{2 n}-1-\varepsilon_{2 n}\right] \ldots}^{D}=g(x)=y, \\
F\left(\sum_{n=1}^{\infty} \frac{\varepsilon_{n}}{d_{1} d_{2} \ldots d_{n}}\right)=\beta_{\varepsilon_{1}, 1}+\sum_{n=2}^{\infty}\left(\beta_{\varepsilon_{n}, n} \prod_{j=1}^{n-1} p_{\varepsilon_{j}, j}\right) .
\end{gathered}
$$

The notion of the shift operator of real number expansion by the positive Cantor series is useful for studying the methods of defining the function $\tilde{F}$.

Definition 2.3. A mapping $\hat{\varphi}$, defined by

$$
\hat{\varphi}(x)=\hat{\varphi}\left(\sum_{n=1}^{\infty} \frac{\varepsilon_{n}}{d_{1} d_{2} \ldots d_{n}}\right)=\sum_{n=2}^{\infty} \frac{\varepsilon_{n}}{d_{2} d_{3} \ldots d_{n}},
$$

is called a shift operator of expansion of the number $x=\Delta_{\varepsilon_{1} \varepsilon_{2} \ldots \varepsilon_{n} \ldots}^{D}$ by the positive Cantor series (1). 
That is, $\hat{\varphi}(x)=d_{1} x-\varepsilon_{1}(x) \equiv d_{1} \Delta_{0 \varepsilon_{2} \varepsilon_{3} \ldots}^{D}$. In addition,

$$
\hat{\varphi}^{k}(x)=\sum_{n=k+1}^{\infty} \frac{\varepsilon_{n}}{d_{k+1} d_{k+2} \ldots d_{n}} \equiv d_{1} d_{2} \ldots d_{k} \underbrace{D \ldots 0}_{k} \varepsilon_{\varepsilon_{k+1} \varepsilon_{k+2} \varepsilon_{k+3} \ldots}^{D}
$$

and

$$
\frac{i}{d_{k}}+\frac{\hat{\varphi}^{k}(x)}{d_{k}}=d_{1} d_{2} \ldots d_{k-1} \Delta_{k-1}^{D} \underbrace{D}_{i \varepsilon_{k+1} \varepsilon_{k+2} \varepsilon_{k+3} \ldots}=\hat{\varphi}^{k-1}(x),
$$

where $i$ is a $k$-th digit in the D-representation (representation by the positive Cantor series) $\Delta_{\varepsilon_{1} \varepsilon_{2} \ldots \varepsilon_{n} \ldots}^{D}$ of $x$.

It is easy to see that the function $\tilde{F}$ is a unique solution of the following infinite functional equations systems (the systems are equivalent because equality (4) holds) in the class of determined and bounded on $[0 ; 1]$ functions:

$$
f\left(\frac{\tilde{i}(x)+\hat{\varphi}^{k}(y)}{d_{k}}\right)=\tilde{\beta}_{i(x), k}+\tilde{p}_{i(x), k} \cdot f\left(\hat{\varphi}^{k}(y)\right),
$$

where $k=1,2, \ldots$, and $i \in A_{d_{k}}, i(x)$ is a $k$-th digit in the nega- $\left(d_{n}\right)$ representation $\Delta_{\varepsilon_{1} \varepsilon_{2} \ldots \varepsilon_{n} \ldots}^{-\left(d_{n}\right)}$ of $x$,

$$
\tilde{i}(x)= \begin{cases}i(x), & \text { if } k \text { is odd } \\ d_{k}-1-i(x), & \text { if } k \text { is even }\end{cases}
$$

$$
f\left(\hat{\varphi}^{k}(y)\right)=\tilde{\beta}_{\varepsilon_{k+1}(x), k+1}+\tilde{p}_{\varepsilon_{k+1}(x), k+1} f\left(\hat{\varphi}^{k+1}(y)\right),
$$

where $k=0,1,2, \ldots$, and $\varepsilon_{k+1} \in A_{d_{k+1}}, y=\Delta_{\varepsilon_{1}\left[d_{2}-1-\varepsilon_{2}\right] \varepsilon_{3} \ldots\left[d_{2 n}-1-\varepsilon_{2 n}\right] \varepsilon_{2 n+1} \ldots}^{D}$. Really,

$$
\tilde{F}(x)=\beta_{\varepsilon_{1}(x), 1}+\sum_{n=2}^{k}\left(\tilde{\beta}_{\varepsilon_{n}(x), n} \prod_{j=1}^{n-1} \tilde{p}_{\varepsilon_{j}(x), j}\right)+\left(\prod_{j=1}^{k} \tilde{p}_{\varepsilon_{j}(x), j}\right) \cdot f\left(\hat{\varphi}^{k}(y)\right),
$$

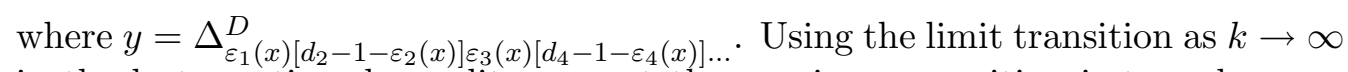
in the last-mentioned equality, we get the proving proposition is true, because the function $\tilde{F}$ is determined and bounded on $[0 ; 1]$ and the third property of the matrix $P$ holds.

The main proposition of the present section is the well-posedness of definition of the function. 
Lemma 3. The values of the function $y=\tilde{F}(x)$ for different representations of nega- $\left(d_{n}\right)$-rational numbers from $[0 ; 1]$ are equal.

P r o o f. Consider a nega- $\left(d_{n}\right)$-rational number $x$ and the following difference:

$$
\begin{aligned}
\delta= & \tilde{F}\left(\Delta_{\varepsilon_{1} \ldots \varepsilon_{n-1} \varepsilon_{n}\left[d_{n+1}-1\right] 0\left[d_{n+3}\right] 0 \ldots}^{-\left(d_{n}\right)}\right)-\tilde{F}\left(\Delta_{\varepsilon_{1} \ldots \varepsilon_{n-1}\left[\varepsilon_{n}-1\right] 0\left[d_{n+2}-1\right] 0\left[d_{n+4}-1\right] 0 \ldots}^{-\left(d_{n}\right)}\right) \\
& =\left(\prod_{j=1}^{n-1} \tilde{p}_{\varepsilon_{j}, j}\right)\left[\left(\tilde{\beta}_{\varepsilon_{n}, n}+\tilde{\beta}_{d_{n+1}-1, n+1} \tilde{p}_{\varepsilon_{n}, n}+\tilde{\beta}_{0, n+2} \tilde{p}_{\varepsilon_{n}, n} \tilde{p}_{d_{n+1}-1, n+1}\right.\right. \\
& \left.+\tilde{\beta}_{d_{n+3}-1, n+3} \tilde{p}_{\varepsilon_{n}, n} \tilde{p}_{d_{n+1}-1, n+1} \tilde{p}_{0, n+2}+\ldots\right)-\left(\tilde{\beta}_{\varepsilon_{n}-1, n}+\tilde{\beta}_{0, n+1} \tilde{p}_{\varepsilon_{n}-1, n}\right. \\
& \left.\left.+\tilde{\beta}_{d_{n+2}-1, n+2} \tilde{p}_{\varepsilon_{n}-1, n} \tilde{p}_{0, n+1}+\tilde{\beta}_{0, n+3} \tilde{p}_{\varepsilon_{n}-1, n} \tilde{p}_{0, n+1} \tilde{p}_{d_{n+2}-1, n+2}+\ldots\right)\right]
\end{aligned}
$$

If $n$ is even, then

$$
\begin{gathered}
\delta=\left(\prod_{j=1}^{n-1} \tilde{p}_{\varepsilon_{j}, j}\right)\left[\beta_{d_{n}-1-\varepsilon_{n}, n}+\beta_{d_{n+1}-1, n+1} p_{d_{n}-1-\varepsilon_{n}, n}\right. \\
\left.+p_{d_{n}-1-\varepsilon_{n}, n} \sum_{k=2}^{\infty}\left(\beta_{d_{n+k}-1, n+k} \prod_{j=1}^{k-1} p_{d_{n+j}-1, n+j}\right)\right]-\left(\prod_{j=1}^{n-1} \tilde{p}_{\varepsilon_{j}, j}\right) \\
\times\left[\beta_{d_{n}-\varepsilon_{n}, n}+\beta_{0, n+1} p_{d_{n}-\varepsilon_{n}, n}+p_{d_{n}-\varepsilon_{n}, n} \sum_{k=2}^{\infty}\left(\beta_{0, n+k} \prod_{j=1}^{k-1} p_{0, n+j}\right)\right] \\
=\left(\prod_{j=1}^{n-1} \tilde{p}_{\varepsilon_{j}, j}\right)\left(-p_{d_{n}-\varepsilon_{n}-1, n}+\left(1-p_{d_{n+1}-1, n+1}\right) p_{d_{n}-\varepsilon_{n}-1, n}\right. \\
\left.+p_{d_{n}-\varepsilon_{n}-1, n} \sum_{k=2}^{\infty}\left[\left(1-p_{d_{n+k}-1, n+k}\right) \prod_{j=1}^{k-1} p_{d_{n+j}-1, n+j}\right]\right)=0 .
\end{gathered}
$$

If $n$ is odd, then

$$
\begin{gathered}
\delta=\left(p_{\varepsilon_{n}-1, n}-\left(1-p_{d_{n+1}-1, n+1}\right) p_{\varepsilon_{n}-1, n}\right. \\
\left.-\left(1-p_{d_{n+2}-1, n+2}\right) p_{\varepsilon_{n}-1, n} p_{d_{n+1}-1, n+1}-\ldots\right) \prod_{j=1}^{n-1} \tilde{p}_{\varepsilon_{j}, j}=0 .
\end{gathered}
$$

Journal of Mathematical Physics, Analysis, Geometry, 2017, vol. 13, No. 1 


\section{Continuity and Monotonicity}

Theorem 3. The function $\tilde{F}$ is:

- continuous;

- monotonic non-decreasing if the elements of the matrix $P$ are non-negative, and strictly increasing if all elements of the matrix $P$ are positive.

P r o of. Continuity. Let $x_{0}=\Delta_{\varepsilon_{1}\left(x_{0}\right) \varepsilon_{2}\left(x_{0}\right) \ldots \varepsilon_{n_{0}}\left(x_{0}\right) \varepsilon_{n_{0}+1}\left(x_{0}\right) \ldots}^{-\left(d_{n}\right)}$ be an arbitrary number from the interval $[0 ; 1]$ and $x=\Delta_{\varepsilon_{1}(x) \varepsilon_{2}(x) \ldots \varepsilon_{n_{0}}(x) \varepsilon_{n_{0}+1}(x) \ldots}^{-\left(d_{n}\right)}$ be a number such that $\varepsilon_{j}(x)=\varepsilon_{j}\left(x_{0}\right)$ for $j=\overline{1, n_{0}-1}$, and $\varepsilon_{n_{0}}(x) \neq \varepsilon_{n_{0}}\left(x_{0}\right)$. Consider the difference

$$
\tilde{F}(x)-\tilde{F}\left(x_{0}\right)=\left(\prod_{j=1}^{n_{0}-1} \tilde{p}_{\varepsilon_{j}\left(x_{0}\right), j}\right)\left(\tilde{F}\left(\hat{\varphi}^{n_{0}-1}(x)\right)-\tilde{F}\left(\hat{\varphi}^{n_{0}-1}\left(x_{0}\right)\right)\right) .
$$

So,

$$
\left|\tilde{F}(x)-\tilde{F}\left(x_{0}\right)\right| \leq\left(\prod_{j=1}^{n_{0}-1}\left|\tilde{p}_{\varepsilon_{j}\left(x_{0}\right), j}\right|\right) \leq\left(\max _{j=1, n_{0}-1}\left|\tilde{p}_{\varepsilon_{j}\left(x_{0}\right), j}\right|\right)^{n_{0}-1} \rightarrow 0 \quad\left(n_{0} \rightarrow \infty\right) .
$$

The last-mentioned condition and $\lim _{x \rightarrow x_{0}} \tilde{F}(x)=\tilde{F}\left(x_{0}\right)$ are equivalent.

Really, the conditions $x \rightarrow x_{0}$ and $n_{0} \rightarrow \infty$ are equivalent for the nega$\left(d_{n}\right)$-irrational number $x_{0}$. It follows that the function $\tilde{F}$ is continuous at each nega- $\left(d_{n}\right)$-irrational point.

Let $x_{0}$ be a nega- $\left(d_{n}\right)$-rational number. In this case, a continuity of the function $\tilde{F}$ at nega- $\left(d_{n}\right)$-rational point $x_{0}$ can be proved by the notion of unilateral borders for the cases of odd and even $n_{0}$.

Monotonicity. Let the elements $p_{i, n}$ of the matrix $P$ be non-negative. It is obvious that

$$
\begin{gathered}
\tilde{F}(0)=\tilde{F}\left(\Delta_{0\left[d_{2}-1\right] 0\left[d_{4}-1\right] \ldots}^{-\left(d_{n}\right)}\right)=\beta_{0,1}+\sum_{n=2}^{\infty}\left(\beta_{0, n} \prod_{j=1}^{n-1} p_{0, j}\right)=\min _{x \in[0 ; 1]} \tilde{F}(x)=0, \\
\tilde{F}(1)=\tilde{F}\left(\Delta_{\left[d_{1}-1\right] 0\left[d_{3}-1\right] 0 \ldots}^{-\left(d_{n}\right)}\right)=\beta_{d_{1}-1,1}+\sum_{n=2}^{\infty}\left(\beta_{d_{n}-1, n} \prod_{j=1}^{n-1} p_{d_{j}-1, j}\right) \\
=\max _{x \in[0 ; 1]} \tilde{F}(x)=1 .
\end{gathered}
$$


Let $x_{1}=\Delta_{\varepsilon_{1}\left(x_{1}\right) \varepsilon_{2}\left(x_{1}\right) \ldots \varepsilon_{n}\left(x_{1}\right) \ldots}^{-\left(d_{n}\right)}$ and $x_{2}=\Delta_{\varepsilon_{1}\left(x_{2}\right) \varepsilon_{2}\left(x_{2}\right) \ldots \varepsilon_{n}\left(x_{2}\right) \ldots}^{-\left(d_{n}\right)}$ be such that $x_{1}<x_{2}$. It is obvious that there exists $n_{0}$ such that $\varepsilon_{j}\left(x_{1}\right)=\varepsilon_{j}\left(x_{2}\right)$ for all $j=\overline{1, n_{0}-1}$, and $\varepsilon_{n_{0}}\left(x_{1}\right)<\varepsilon_{n_{0}}\left(x_{2}\right)$ in the case of an odd $n_{0}$, or $\varepsilon_{n_{0}}\left(x_{1}\right)>$ $\varepsilon_{n_{0}}\left(x_{2}\right)$ in the case of an even $n_{0}$.

Thus,

$$
\begin{aligned}
\tilde{F}\left(x_{2}\right) & -\tilde{F}\left(x_{1}\right)=\left(\prod_{j=1}^{n_{0}-1} \tilde{p}_{\varepsilon_{j}\left(x_{2}\right), j}\right)\left(\tilde{\beta}_{\varepsilon_{n_{0}}\left(x_{2}\right), n_{0}}-\tilde{\beta}_{\varepsilon_{n_{0}}\left(x_{1}\right), n_{0}}\right. \\
& +\sum_{m=1}^{\infty}\left(\tilde{\beta}_{\varepsilon_{n_{0}+m}\left(x_{2}\right), n_{0}+m} \prod_{j=0}^{m-1} \tilde{p}_{\varepsilon_{n_{0}+j}\left(x_{2}\right), n_{0}+j}\right) \\
& \left.-\sum_{m=1}^{\infty}\left(\tilde{\beta}_{\varepsilon_{n_{0}+m}\left(x_{1}\right), n_{0}+m} \prod_{j=0}^{m-1} \tilde{p}_{\varepsilon_{n_{0}+j}\left(x_{1}\right), n_{0}+j}\right)\right) .
\end{aligned}
$$

Since

$$
\begin{aligned}
\kappa & =\sum_{m=1}^{\infty}\left(\tilde{\beta}_{\varepsilon_{n_{0}+m}\left(x_{2}\right), n_{0}+m} \prod_{j=0}^{m-1} \tilde{p}_{\varepsilon_{n_{0}+j}\left(x_{2}\right), n_{0}+j}\right) \\
& -\sum_{m=1}^{\infty}\left(\tilde{\beta}_{\varepsilon_{n_{0}+m}\left(x_{1}\right), n_{0}+m} \prod_{j=0}^{m-1} \tilde{p}_{\varepsilon_{n_{0}+j}\left(x_{1}\right), n_{0}+j}\right) \\
\geq & -\sum_{m=1}^{\infty}\left(\tilde{\beta}_{\varepsilon_{n_{0}+m}\left(x_{1}\right), n_{0}+m} \prod_{j=0}^{m-1} \tilde{p}_{\varepsilon_{n_{0}+j}\left(x_{1}\right), n_{0}+j}\right),
\end{aligned}
$$

where in the case of an odd $n_{0}$

$$
\begin{gathered}
\kappa \geq-p_{\varepsilon_{n_{0}}\left(x_{1}\right), n_{0}}\left(1-p_{d_{n_{0}+1}-1, n_{0}+1}+\sum_{m=2}^{\infty}\left[\left(1-p_{d_{n_{0}+m}-1, n_{0}+m}\right) \prod_{j=1}^{m-1} p_{d_{n_{0}+j}-1, n_{0}+j}\right]\right) \\
=-p_{\varepsilon_{n_{0}}\left(x_{1}\right), n_{0}},
\end{gathered}
$$

for the case of an even $n_{0}$ we have

$$
\kappa \geq-p_{d_{n_{0}}-1-\varepsilon_{n_{0}}\left(x_{1}\right), n_{0}}\left(\max _{x \in[0,1]} \tilde{F}\left(\hat{\varphi}^{n_{0}}\left(x_{1}\right)\right)\right)=-p_{d_{n_{0}}-1-\varepsilon_{n_{0}}\left(x_{1}\right), n_{0}} .
$$

Hence, if $n_{0}$ is odd, then

$$
\tilde{F}\left(x_{2}\right)-\tilde{F}\left(x_{1}\right)=\left(\prod_{j=1}^{n_{0}-1} \tilde{p}_{\varepsilon_{j}\left(x_{2}\right), j}\right)\left(\tilde{\beta}_{\varepsilon_{n_{0}}\left(x_{2}\right), n_{0}}-\tilde{\beta}_{\varepsilon_{n_{0}}\left(x_{1}\right), n_{0}}+\kappa\right)
$$

Journal of Mathematical Physics, Analysis, Geometry, 2017, vol. 13, No. 1 
$\geq\left(\prod_{j=1}^{n_{0}-1} \tilde{p}_{\varepsilon_{j}\left(x_{2}\right), j}\right)\left(p_{\varepsilon_{n_{0}}\left(x_{1}\right), n_{0}}+p_{\varepsilon_{n_{0}}\left(x_{1}\right)+1, n_{0}}+\ldots+p_{\varepsilon_{n_{0}}\left(x_{2}\right)-1, n_{0}}-p_{\varepsilon_{n_{0}}\left(x_{1}\right), n_{0}}\right) \geq 0$.

If $n_{0}$ is even, then

$$
\begin{gathered}
\tilde{F}\left(x_{2}\right)-\tilde{F}\left(x_{1}\right)=\left(\prod_{j=1}^{n_{0}-1} \tilde{p}_{\varepsilon_{j}\left(x_{2}\right), j}\right) \cdot\left(\tilde{\beta}_{\varepsilon_{n_{0}}\left(x_{2}\right), n_{0}}-\tilde{\beta}_{\varepsilon_{n_{0}}\left(x_{1}\right), n_{0}}+\kappa\right) \\
=\left(\prod_{i=1}^{n_{0}-1} \tilde{p}_{i, \varepsilon_{i}\left(x_{2}\right)}\right)\left(p_{d_{n_{0}}-1-\varepsilon_{n_{0}}\left(x_{1}\right), n_{0}}+p_{d_{n_{0}}-\varepsilon_{n_{0}}\left(x_{1}\right), n_{0}}+\ldots\right. \\
\left.+p_{d_{n_{0}}-2-\varepsilon_{n_{0}}\left(x_{2}\right), n_{0}}-p_{d_{n_{0}}-1-\varepsilon_{n_{0}}\left(x_{1}\right), n_{0}}\right) \geq 0 .
\end{gathered}
$$

It is easy to see that the condition $\tilde{F}\left(x_{2}\right)-\tilde{F}\left(x_{1}\right)>0$ holds if all elements $p_{i, n}$ of the matrix $P$ are positive.

Let the elements $p_{i, n}$ of $P$ be non-negative.

Let $\eta$ be a random variable defined by the Cantor expansion

$$
\eta=\frac{\xi_{1}}{d_{1}}+\frac{\xi_{2}}{d_{1} d_{2}}+\frac{\xi_{3}}{d_{1} d_{2} d_{3}}+\ldots+\frac{\xi_{k}}{d_{1} d_{2} \ldots d_{k}}+\ldots \equiv \Delta_{\xi_{1} \xi_{2} \ldots \xi_{k} \ldots}^{D}
$$

where

$$
\xi_{k}= \begin{cases}\varepsilon_{k}, & \text { if } k \text { is odd } \\ d_{k}-1-\varepsilon_{k}, & \text { if } k \text { is even, }\end{cases}
$$

and the digits $\xi_{k}(k=1,2,3, \ldots)$ are random and take the values $0,1, \ldots, d_{k}-1$ with probabilities $p_{0, k}, p_{1, k}, \ldots, p_{d_{k}-1, k}$. That is, $\xi_{k}$ are independent, and $P\left\{\xi_{k}=\right.$ $\left.i_{k}\right\}=p_{i_{k}, k}, i_{k} \in A_{d_{k}}$.

From the definition of the distribution function and the expressions

$$
\begin{gathered}
\{\eta<x\}=\left\{\xi_{1}<\varepsilon_{1}(x)\right\} \cup\left\{\xi_{1}=\varepsilon_{1}(x), \xi_{2}<d_{2}-1-\varepsilon_{2}(x)\right\} \cup \ldots \\
\cup\left\{\xi_{1}=\varepsilon_{1}(x), \xi_{2}=d_{2}-1-\varepsilon_{2}(x), \ldots, \xi_{2 k-1}<\varepsilon_{2 k-1}(x)\right\} \\
\cup\left\{\xi_{1}=\varepsilon_{1}(x), \xi_{2}=d_{2}-1-\varepsilon_{2}(x), \ldots, \xi_{2 k-1}=\varepsilon_{2 k-1}(x), \xi_{2 k}<d_{2 k}-1-\varepsilon_{2 k}(x)\right\} \cup \ldots, \\
P\left\{\xi_{1}=\varepsilon_{1}(x), \xi_{2}=d_{2}-1-\varepsilon_{2}(x), \ldots, \xi_{2 k-1}<\varepsilon_{2 k-1}(x)\right\} \\
=\beta_{\varepsilon_{2 k-1}(x), 2 k-1} \prod_{j=1}^{2 k-2} \tilde{p}_{\varepsilon_{j}(x), j}
\end{gathered}
$$

and

$$
P\left\{\xi_{1}=\varepsilon_{1}(x), \xi_{2}=d_{2}-1-\varepsilon_{2}(x), \ldots, \xi_{2 k}<d_{2 k}-1-\varepsilon_{2 k}(x)\right\}
$$




$$
=\beta_{d_{2 k}-1-\varepsilon_{2 k}(x), 2 k} \prod_{j=1}^{2 k-1} \tilde{p}_{\varepsilon_{j}(x), j},
$$

it is easy to see that the following proposition is a corollary of the last-mentioned theorem.

Corollary 3.1. The distribution function $\tilde{F}_{\eta}$ of the random variable $\eta$ has the form

$$
\tilde{F}_{\eta}(x)= \begin{cases}0, & x<0 ; \\ \beta_{\varepsilon_{1}(x), 1}+\sum_{k=2}^{\infty}\left[\tilde{\beta}_{\varepsilon_{k}(x), k} \prod_{j=1}^{k-1} \tilde{p}_{\varepsilon_{j}(x), j}\right], & 0 \leq x<1 ; \\ 1, & x \geq 1,\end{cases}
$$

where $\tilde{p}_{\varepsilon_{j(x), j}} \geq 0$.

\section{Integral Properties}

Theorem 4. The Lebesgue integral of the function $\tilde{F}$ can be calculated by the formula

$$
\int_{0}^{1} \tilde{F}(x) d x=\sum_{n=1}^{\infty} \frac{\tilde{\beta}_{0, n}+\tilde{\beta}_{1, n}+\tilde{\beta}_{2, n}+\ldots+\tilde{\beta}_{d_{n}-1, n}}{d_{1} d_{2} \ldots d_{n}} .
$$

P r o o f. Denote $y=g(x)$ (the function $g$ was defined in Section 2.). Using the definition of $\tilde{F}$ (and the properties of $\tilde{F}$ that follow from different ways of defining the function) and the properties of the Lebesgue integral, we have

$$
\begin{gathered}
\int_{0}^{1} \tilde{F}(x) d x=\int_{0}^{\frac{1}{d_{1}}} F(y) d y+\int_{\frac{1}{d_{1}}}^{\frac{2}{d_{1}}} F(y) d y+\ldots+\int_{\frac{d_{1}-1}{d_{1}}}^{1} F(y) d y \\
=\int_{0}^{\frac{1}{d_{1}}} p_{0,1} F(\hat{\varphi}(y)) d y+\int_{\frac{1}{d_{1}}}^{\frac{2}{d_{1}}}\left[p_{0,1}+p_{1,1} F(\hat{\varphi}(y))\right] d y \\
+\int_{\frac{2}{d_{1}}}^{\frac{3}{d_{1}}}\left[\beta_{2,1}+p_{2,1} F(\hat{\varphi}(y))\right] d y+\ldots+\int_{\frac{d_{1}-1}{d_{1}}}^{1}\left[\beta_{d_{1}-1,1}+p_{d_{1}-1,1} F(\hat{\varphi}(y))\right] d y .
\end{gathered}
$$

Since $y=\frac{\varepsilon_{1}}{d_{1}}+\frac{1}{d_{1}} \hat{\varphi}(y)$ and $d y=\frac{1}{d_{1}} d(\hat{\varphi}(y))$, then

$$
\int_{0}^{1} \tilde{F}(x) d x=\frac{p_{0,1}}{d_{1}} \int_{0}^{1} F(\hat{\varphi}(y)) d(\hat{\varphi}(y))+\left.\beta_{1,1} y\right|_{\frac{1}{d_{1}}} ^{\frac{2}{d_{1}}}+\frac{p_{1,1}}{d_{1}} \int_{0}^{1} F(\hat{\varphi}(y)) d(\hat{\varphi}(y))+\left.\beta_{2,1} y\right|_{\frac{2}{d_{1}}} ^{\frac{3}{d_{1}}}
$$




$$
\begin{gathered}
+\frac{p_{2,1}}{d_{1}} \int_{0}^{1} F(\hat{\varphi}(y)) d(\hat{\varphi}(y))+\ldots+\left.\beta_{d_{1}-1,1} y\right|_{\frac{d_{1}-1}{d_{1}}} ^{1}+\frac{p_{d_{1}-1,1}}{d_{1}} \int_{0}^{1} F(\hat{\varphi}(y)) d(\hat{\varphi}(y)) \\
=\frac{\beta_{1,1}+\beta_{2,1}+\ldots+\beta_{d_{1}-1,1}}{d_{1}}+\frac{1}{d_{1}} \int_{0}^{1} F(\hat{\varphi}(y)) d(\hat{\varphi}(y))
\end{gathered}
$$

Analogously, from the relation between D-representation and nega- $\left(d_{n}\right)$-representation, it follows that

$$
\begin{gathered}
\int_{0}^{1} F(\hat{\varphi}(y)) d(\hat{\varphi}(y))=\int_{\frac{d_{2}-1}{d_{2}}}^{1} p_{0,2} F\left(\hat{\varphi}^{2}(y)\right) d(\hat{\varphi}(y)) \\
+\int_{\frac{d_{2}-2}{d_{2}}}^{\frac{d_{2}-1}{d_{2}}}\left[\beta_{1,2}+p_{1,2} F\left(\hat{\varphi}^{2}(y)\right)\right] d(\hat{\varphi}(y))+\ldots+\int_{0}^{\frac{1}{d_{2}}}\left[\beta_{d_{2}-1,2}+p_{d_{2}-1,2} F\left(\hat{\varphi}^{2}(y)\right)\right] d(\hat{\varphi}(y)) .
\end{gathered}
$$

Since $\hat{\varphi}(y)=\frac{d_{2}-1-\varepsilon_{2}}{d_{2}}+\frac{1}{d_{2}} \hat{\varphi}^{2}(y)$ and $d(\hat{\varphi}(y))=\frac{1}{d_{2}} d\left(\hat{\varphi}^{2}(y)\right)$, we obtain

$$
\begin{gathered}
\int_{0}^{1} F(\hat{\varphi}(y)) d(\hat{\varphi}(y))=\frac{p_{0,2}}{d_{2}} \int_{0}^{1} F\left(\hat{\varphi}^{2}(y)\right) d\left(\hat{\varphi}^{2}(y)\right)+\left.\beta_{1,2} y\right|_{\frac{d_{2}-2}{d_{2}}} ^{\frac{d_{2}-1}{d_{2}}} \\
+\frac{p_{1,2}}{d_{2}} \int_{0}^{1} F\left(\hat{\varphi}^{2}(y)\right) d\left(\hat{\varphi}^{2}(y)\right)+\ldots+\left.\beta_{d_{2}-1,2} y\right|_{0} ^{\frac{1}{d_{2}}}+\frac{p_{d_{2}-1,2}}{d_{2}} \int_{0}^{1} F\left(\hat{\varphi}^{2}(y)\right) d\left(\hat{\varphi}^{2}(y)\right) \\
=\frac{\beta_{1,2}+\beta_{2,2}+\ldots+\beta_{d_{2}-1,2}}{d_{2}}+\frac{1}{d_{2}} \int_{0}^{1} F\left(\hat{\varphi}^{2}(y)\right) d\left(\hat{\varphi}^{2}(y)\right) .
\end{gathered}
$$

So,

$$
\begin{gathered}
\int_{0}^{1} \tilde{F}(x) d x=\frac{\beta_{1,1}+\beta_{2,1}+\ldots+\beta_{d_{1}-1,1}}{d_{1}} \\
+\frac{\beta_{1,2}+\beta_{2,2}+\ldots+\beta_{d_{2}-1,2}}{d_{1} d_{2}}+\frac{1}{d_{1} d_{2}} \int_{0}^{1} F\left(\hat{\varphi}^{2}(y)\right) d\left(\hat{\varphi}^{2}(y)\right) .
\end{gathered}
$$


Analogously,

$$
\begin{gathered}
\int_{0}^{1} \tilde{F}(x) d x=\sum_{j=1}^{n} \frac{\tilde{\beta}_{0, j}+\tilde{\beta}_{1, j}+\tilde{\beta}_{2, j}+\ldots+\tilde{\beta}_{d_{j}-1, j}}{d_{1} d_{2} \ldots d_{j}} \\
\quad+\frac{1}{d_{1} d_{2} \ldots d_{n}} \int_{0}^{1} F\left(\hat{\varphi}^{n}(y)\right) d\left(\hat{\varphi}^{n}(y)\right) .
\end{gathered}
$$

Continuing the process indefinitely, we obtain

$$
\int_{0}^{1} \tilde{F}(x) d x=\sum_{n=1}^{\infty} \frac{\tilde{\beta}_{0, n}+\tilde{\beta}_{1, n}+\tilde{\beta}_{2, n}+\ldots+\tilde{\beta}_{d_{n}-1, n}}{d_{1} d_{2} \ldots d_{n}} .
$$

\section{Self-affine Properties}

Theorem 5. If the elements $p_{i, n}$ of the matrix $P$ are positive, then the graph $\Gamma_{\tilde{F}}$ of the function $\tilde{F}$ in the space $\mathbb{R}^{2}$ is the set

$$
\Gamma_{\tilde{F}}=\bigcup_{x \in[0 ; 1]}\left(x ; \ldots \circ \psi_{\varepsilon_{n}, n} \circ \ldots \circ \psi_{\varepsilon_{2}, 2} \circ \psi_{\varepsilon_{1}, 1}(x)\right),
$$

where $x=\Delta_{\varepsilon_{1}\left[d_{2}-1-\varepsilon_{2}\right] \varepsilon_{3}\left[d_{4}-1-\varepsilon_{4}\right] \ldots}^{D}$,

$$
\begin{gathered}
\psi_{i_{n}, n}:\left\{\begin{array}{l}
x^{\prime}=\frac{1}{d_{n}} x+\frac{\omega_{i_{n}, n}}{d_{n}} ; \\
y^{\prime}=\tilde{\beta}_{i_{n}, n}+\tilde{p}_{i_{n}, n} y,
\end{array}\right. \\
\omega_{i_{n}, n}= \begin{cases}i_{n}, & \text { if } n \text { is odd } ; \\
d_{n}-1-i_{n}, & \text { if } n \text { is even },\end{cases}
\end{gathered}
$$

$i_{n} \in A_{d_{n}}$.

P r o o f. Since the following expressions

$$
\begin{gathered}
f(x)=\beta_{i, 1}+p_{i, 1} f(\hat{\varphi}(x)), \\
f\left(\frac{i+x}{d_{1}}\right)=\beta_{i, 1}+p_{i, 1} f(x)
\end{gathered}
$$


are equivalent for $x=\Delta_{\varepsilon_{1}\left[d_{2}-1-\varepsilon_{2}\right] \varepsilon_{3}\left[d_{4}-1-\varepsilon_{4}\right] \ldots}^{D}$, it is obvious that

$$
\psi_{i_{1}, 1}:\left\{\begin{array}{l}
x^{\prime}=\frac{1}{d_{1}} x+\frac{i_{1}}{d_{1}} ; \\
y^{\prime}=\beta_{i_{1}, 1}+p_{i_{1}, 1} y .
\end{array}\right.
$$

Consider the affine transformations $\psi_{i, 2}, i=\overline{0, d_{2}-1}$. Since the expressions

$$
\begin{gathered}
f(\hat{\varphi}(x))=\beta_{d_{2}-1-i, 2}+p_{d_{2}-1-i, 2} f\left(\hat{\varphi}^{2}(x)\right), \\
f\left(\frac{d_{2}-1-i+\hat{\varphi}(x)}{d_{2}}\right)=\beta_{d_{2}-1-i, 2}+p_{d_{2}-1-i, 2} f(\hat{\varphi}(x))
\end{gathered}
$$

are equivalent, we have

$$
\psi_{i_{2}, 2}:\left\{\begin{array}{l}
x^{\prime}=\frac{1}{d_{2}} x+\frac{d_{2}-1-i_{2}}{d_{2}} ; \\
y^{\prime}=\beta_{d_{2}-1-i_{2}, 2}+p_{d_{2}-1-i_{2}, 2} y .
\end{array}\right.
$$

By induction, we obtain

$$
\psi_{i_{n}, n}:\left\{\begin{aligned}
x^{\prime} & =\frac{1}{d_{n}} x+\frac{\omega_{i_{n}, n}}{d_{n}} \\
y^{\prime} & =\tilde{\beta}_{i_{n}, n}+\tilde{p}_{i_{n}, n} y .
\end{aligned}\right.
$$

So,

$$
\bigcup_{x \in[0 ; 1]}\left(x ; \ldots \circ \psi_{\varepsilon_{n}, n} \circ \ldots \circ \psi_{\varepsilon_{2}, 2} \circ \psi_{\varepsilon_{1}, 1}(x)\right) \equiv G \subset \Gamma_{\tilde{F}} .
$$

Let $T\left(x_{0}, \tilde{F}\left(x_{0}\right)\right) \in \Gamma_{\tilde{F}} . \quad$ Consider a point $x_{n}=\hat{\varphi}^{n}\left(x_{0}\right)$, where $x_{0}=$ $\Delta_{\varepsilon_{1}\left[d_{2}-1-\varepsilon_{2}\right] \varepsilon_{3}\left[d_{4}-1-\varepsilon_{4}\right] \ldots}^{D}$ is a fixed point from $[0 ; 1]$.

Since for any $n \in \mathbb{N}, \varepsilon_{n}$ and $d_{n}-1-\varepsilon_{n}$ belong to $A_{d_{n}}$,

$$
f\left(\hat{\varphi}^{k}\left(x_{0}\right)\right)=\tilde{\beta}_{\varepsilon_{k+1}, k+1}+\tilde{p}_{\varepsilon_{k+1}, k+1} f\left(\hat{\varphi}^{k+1}\left(x_{0}\right)\right), k=0,1, \ldots
$$

and from $\bar{T}\left(\hat{\varphi}^{k}\left(x_{0}\right) ; \tilde{F}\left(\hat{\varphi}^{k}\left(x_{0}\right)\right)\right) \in \Gamma_{\tilde{F}}$, it follows that

$$
\psi_{i_{k}, k} \circ \ldots \circ \psi_{i_{2}, 2} \circ \psi_{i_{1}, 1}(\bar{T})=T_{0}\left(x_{0} ; \tilde{F}\left(x_{0}\right)\right) \in \Gamma_{\tilde{F}}, \quad i_{k} \in A_{d_{k}}, \quad k \rightarrow \infty .
$$

Therefore, $\Gamma_{\tilde{F}} \subset G$, and thus

$$
\Gamma_{\tilde{F}}=\bigcup_{x \in[0 ; 1]}\left(x ; \ldots \circ \psi_{\varepsilon_{n}, n} \circ \ldots \circ \psi_{\varepsilon_{2}, 2} \circ \psi_{\varepsilon_{1}, 1}(x)\right) .
$$




\section{Differential Properties when the Elements of the Matrix $P$ are Non-negative}

Let the elements $p_{i, n}$ of the matrix $P$ be non-negative.

Definition 6.4. Let $c_{1}, c_{2}, \ldots, c_{n}$ be an ordered set of integer numbers such that $c_{i} \in A_{d_{i}}$ for all $i=\overline{1, n}$.

A cylinder $\Delta_{c_{1} c_{2} \ldots c_{n}}^{-\left(d_{n}\right)}$ of rank $n$ with the base $c_{1} c_{2} \ldots c_{n}$ is called a set of all numbers from $[0 ; 1]$ such that the first $n$ digits of the nega- $\left(d_{n}\right)$-representation of the numbers are equal to $c_{1}, c_{2}, \ldots, c_{n}$. That is,

$$
\Delta_{c_{1} c_{2} \ldots c_{n}}^{-\left(d_{n}\right)} \equiv\left\{x: x=\Delta_{c_{1} c_{2} \ldots c_{n} \varepsilon_{n+1} \ldots \varepsilon_{n+k} \ldots}^{-\left(d_{n}\right)}, \varepsilon_{n+k} \in A_{d_{n+k}}\right\}
$$

Definition 6.5. The change $\mu_{\tilde{F}}$ in the function $\tilde{F}$ on the cylinder $\Delta_{c_{1} c_{2} \ldots c_{n}}^{-\left(d_{n}\right)}$ is called a value $\mu_{\tilde{F}}\left(\Delta_{c_{1} c_{2} \ldots c_{n}}^{-\left(d_{n}\right)}\right)$ defined by the equality

$$
\mu_{\tilde{F}}\left(\Delta_{c_{1} c_{2} \ldots c_{n}}^{-\left(d_{n}\right)}\right)=\tilde{F}\left(\sup \Delta_{c_{1} c_{2} \ldots c_{n}}^{-\left(d_{n}\right)}\right)-\tilde{F}\left(\inf \Delta_{c_{1} c_{2} \ldots c_{n}}^{-\left(d_{n}\right)}\right) .
$$

Lemma 4. The following equalities are true:

1.

$$
\mu_{\tilde{F}}\left(\Delta_{c_{1} c_{2} \ldots c_{n}}^{-\left(d_{n}\right)}\right)=\prod_{j=1}^{n} \tilde{p}_{c_{j}, j} \geq 0 .
$$

2. Let $x_{0}=\Delta_{\varepsilon_{1} \varepsilon_{2} \ldots \varepsilon_{n} \ldots}^{-\left(d_{n}\right)}$ be a nega- $\left(d_{n}\right)$-irrational point, then

$$
\tilde{F}^{\prime}\left(x_{0}\right)=\lim _{n \rightarrow \infty}\left(\prod_{j=1}^{n} d_{j} \tilde{p}_{\varepsilon_{j}, j}\right) .
$$

P r o o f.

1. Calculate the change $\mu_{\tilde{F}}$ in the function $\tilde{F}$ on the cylinders $\Delta_{c_{1} c_{2} \ldots c_{n}}^{-\left(d_{n}\right)}$. That is, on the following closed intervals:

$$
\begin{gathered}
{\left[\Delta_{c_{1} c_{2} \ldots c_{2 n-1}\left[d_{2 n}-1\right] 0\left[d_{2 n+2}-1\right] 0\left[d_{2 n+4}-1\right] \ldots}^{-\left(d_{n}\right)} ; \Delta_{\left.c_{1} c_{2} \ldots c_{2 n-1} 0\left[d_{2 n+1}-1\right] 0\left[d_{2 n+3}-1\right] \ldots\right]}^{-\left(d_{n}\right)}\right.} \\
{\left[\Delta_{c_{1} c_{2} \ldots c_{2 n} 0\left[d_{2 n+2}-1\right] 0\left[d_{2 n+4}-1\right] \ldots}^{-\left(d_{n}\right)} ; \Delta_{\left.c_{1} c_{2} \ldots c_{2 n}\left[d_{2 n+1}-1\right] 0\left[d_{2 n+3}-1\right] 0\left[d_{2 n+5}-1\right] \ldots\right]}^{-\left(d_{n}\right)}\right.} \\
\mu_{\tilde{F}}\left(\Delta_{c_{1} c_{2} \ldots c_{2 n-1}}^{-\left(d_{n}\right)}\right)=\tilde{F}\left(\Delta_{c_{1} c_{2} \ldots c_{2 n-1} 0\left[d_{2 n+1}-1\right] 0\left[d_{2 n+3}-1\right] \ldots}^{-\left(d_{n}\right)}\right)
\end{gathered}
$$




$$
\begin{gathered}
-\tilde{F}\left(\Delta_{c_{1} c_{2} \ldots c_{2 n-1}\left[d_{2 n}-1\right] 0\left[d_{2 n+2}-1\right] 0\left[d_{2 n+4}-1\right] \ldots}^{-\left(d_{n}\right)}\right)=\left(\prod_{j=1}^{2 n-1} \tilde{p}_{c_{j}, j}\right) \\
\times\left(\beta_{d_{2 n}-1,2 n}+\beta_{d_{2 n+1}-1,2 n+1} p_{d_{2 n}-1,2 n}+\beta_{d_{2 n+2}-1,2 n+2} p_{d_{2 n}-1,2 n} p_{d_{2 n+1}-1,2 n+1}+\ldots\right) \\
=\left(\prod_{j=1}^{2 n-1} \tilde{p}_{c_{j}, j}\right)\left(1-p_{d_{2 n}-1,2 n}+\left(1-p_{d_{2 n+1}-1,2 n+1}\right) p_{d_{2 n}-1,2 n}\right. \\
\left.+\left(1-p_{d_{2 n+2}-1,2 n+2}\right) p_{d_{2 n}-1,2 n} p_{d_{2 n+1}-1,2 n+1}+\ldots\right)=\left(\prod_{j=1}^{2 n-1} \tilde{p}_{c_{j}, j}\right) .
\end{gathered}
$$

Analogously,

$$
\begin{gathered}
\mu_{\tilde{F}}\left(\Delta_{c_{1} c_{2} \ldots c_{2 n}}^{-\left(d_{n}\right)}\right)=\tilde{F}\left(\Delta_{c_{1} c_{2} \ldots c_{2 n}\left[d_{2 n+1}-1\right] 0\left[d_{2 n+3}-1\right] 0\left[d_{2 n+5}-1\right] \ldots}^{-\left(d_{n}\right)}\right) \\
-\tilde{F}\left(\Delta_{c_{1} c_{2} \ldots c_{2 n} 0\left[d_{2 n+2}-1\right] 0\left[d_{2 n+4}-1\right] \ldots}^{-\left(d_{n}\right)}\right) \\
=\left(\prod_{j=1}^{2 n} \tilde{p}_{c_{j}, j}\right)\left(\beta_{d_{2 n+1}-1,2 n+1}+\beta_{d_{2 n+2}-1,2 n+2} p_{d_{2 n+1}-1,2 n+1}\right. \\
\left.+\beta_{d_{2 n+3}-1,2 n+3} p_{d_{2 n+1}-1,2 n+1} p_{d_{2 n+2}-1,2 n+2}+\ldots\right)=\left(\prod_{j=1}^{2 n} \tilde{p}_{c_{j}, j}\right) .
\end{gathered}
$$

So,

$$
\mu_{\tilde{F}}\left(\Delta_{c_{1} c_{2} \ldots c_{n}}^{-\left(d_{n}\right)}\right)=\left(\prod_{j=1}^{n} \tilde{p}_{c_{j}, j}\right) \geq 0 .
$$

2. Find the derivative of $\tilde{F}$ at the nega- $\left(d_{n}\right)$-irrational point $x_{0}=\Delta_{\varepsilon_{1} \varepsilon_{2} \ldots \varepsilon_{n} \ldots}^{-\left(d_{n}\right)}$. Since

$$
x_{0}=\Delta_{\varepsilon_{1} \varepsilon_{2} \ldots \varepsilon_{n} \ldots}^{-\left(d_{n}\right)}=\bigcap_{n=1}^{\infty} \Delta_{\varepsilon_{1} \varepsilon_{2} \ldots \varepsilon_{n}}^{-\left(d_{n}\right)},
$$

we have

$$
\begin{aligned}
\tilde{F}^{\prime}\left(x_{0}\right) & =\lim _{n \rightarrow \infty} \frac{\mu_{\tilde{F}}\left(\Delta_{\varepsilon_{1} \varepsilon_{2} \ldots \varepsilon_{n}}^{-\left(d_{n}\right)}\right)}{\left|\Delta_{\varepsilon_{1} \varepsilon_{2} \ldots \varepsilon_{n}}^{-\left(d_{n}\right)}\right|}=\lim _{n \rightarrow \infty} \frac{\prod_{j=1}^{n} \tilde{p}_{\varepsilon_{j}, j}}{\frac{1}{d_{1} d_{2} \ldots d_{n}}} \\
= & \lim _{n \rightarrow \infty}\left(\prod_{j=1}^{n} d_{j} \tilde{p}_{\varepsilon_{j}, j}\right)=\prod_{j=1}^{\infty}\left(d_{j} \tilde{p}_{\varepsilon_{j}, j}\right) .
\end{aligned}
$$


Since the function under consideration is continuous and monotonic (by the Lebesgue theorem), it has a finite derivative almost everywhere in the sense of the Lebesgue measure. But $\tilde{F}^{\prime}\left(x_{0}\right)=\infty$ in the case when the condition $a_{n}=$ $d_{n} \tilde{p}_{\varepsilon_{n}, n}>1$ holds for all positive integers $n$ except perhaps a finite number of $n$. Therefore,

- if $a_{n} \geq 1$ holds for a finite set of values $n$, then $\tilde{F}^{\prime}\left(x_{0}\right)=0$;

- if $a_{n}=1$ for all $n \in \mathbb{N}$ (it is true only for $\tilde{F}(x)=x$ ), then $\tilde{F}^{\prime}\left(x_{0}\right)=1$;

- if $p_{\varepsilon_{n}, n} \neq \frac{1}{d_{n}}$ holds only for a finite set of values $n$, then $0 \leq \tilde{F}^{\prime}\left(x_{0}\right)<\infty$.

\section{Nondifferentiable Functions}

Let $p_{i, n} \in(-1 ; 1)$ for all $n \in \mathbb{N}, i=\overline{0, d_{n}-1}$.

In this case, it follows from the statement 1 of Lemma 4 that the function $\tilde{F}$ does not have any arbitrary small monotonicity interval if for each $n \in \mathbb{N}$ the numbers $p_{i, n}$, where $i=\overline{0, d_{n}-1}$, are either non-negative or negative.

Theorem 6. Let $p_{\varepsilon_{n}, n} p_{\varepsilon_{n}-1, n}<0$ for all $n \in \mathbb{N}, \varepsilon_{n} \in A_{d_{n}} \backslash\{0\}$, and the conditions

$$
\lim _{n \rightarrow \infty} \prod_{k=1}^{n} d_{k} p_{0, k} \neq 0, \lim _{n \rightarrow \infty} \prod_{k=1}^{n} d_{k} p_{d_{k}-1, k} \neq 0
$$

hold simultaneously. Then the function $\tilde{F}$ is nowhere differentiable on $[0 ; 1]$.

P r o o f. Choose some nega- $\left(d_{n}\right)$-rational point $x_{0}$ :

$$
x_{0}=\Delta_{\varepsilon_{1} \varepsilon_{2} \ldots \varepsilon_{n-1} \varepsilon_{n}\left[d_{n+1}-1\right] 0\left[d_{n+3}-1\right] \ldots}^{-\left(d_{n}\right)}=\Delta_{\varepsilon_{1} \varepsilon_{2} \ldots \varepsilon_{n-1}\left[\varepsilon_{n}-1\right] 0\left[d_{n+2}-1\right] 0\left[d_{n+4}-1\right] \ldots}^{-\left(d_{n}\right)},
$$

where $\varepsilon_{n} \neq 0$.

Let us introduce some notations. Let $n$ be odd, then

$x_{0}=x_{0}^{(1)}=\Delta_{\varepsilon_{1} \varepsilon_{2} \ldots \varepsilon_{n-1} \varepsilon_{n}\left[d_{n+1}-1\right] 0\left[d_{n+3}-1\right] \ldots}^{-\left(d_{n}\right)}=\Delta_{\varepsilon_{1} \varepsilon_{2} \ldots \varepsilon_{n-1}\left[\varepsilon_{n}-1\right] 0\left[d_{n+2}-1\right] 0\left[d_{n+4}-1\right] \ldots}^{-\left(d_{n}\right)}=x_{0}^{(2)}$

and

$x_{0}=x_{0}^{(1)}=\Delta_{\varepsilon_{1} \varepsilon_{2} \ldots \varepsilon_{n-1}\left[\varepsilon_{n}-1\right] 0\left[d_{n+2}-1\right] 0\left[d_{n+4}-1\right] \ldots}^{-\left(d_{n}\right)}=\Delta_{\varepsilon_{1} \varepsilon_{2} \ldots \varepsilon_{n-1} \varepsilon_{n}\left[d_{n+1}-1\right] 0\left[d_{n+3}-1\right] \ldots}^{-\left(d_{n}\right)}=x_{0}^{(2)}$

in the case of even number $n$. 
Let us consider the sequences $\left(x_{k}^{\prime}\right),\left(x_{k}^{\prime \prime}\right)$ such that

$$
\begin{aligned}
& x_{k}^{\prime}=\left\{\begin{array}{l}
\Delta_{\varepsilon_{1} \ldots \varepsilon_{n-1} \varepsilon_{n}\left[d_{n+1}-1\right] 0\left[d_{n+3}-1\right] 0 \ldots\left[d_{n+k-1}-1\right] 1\left[d_{n+k+1}-1\right] 0\left[d_{n+k+3}-1\right] \ldots}^{-\left(d_{n}\right)} \\
n \in \mathbb{O}, k \in \mathbb{E} ; \\
\Delta_{\varepsilon_{1} \ldots \varepsilon_{n-1} \varepsilon_{n}\left[d_{n+1}-1\right] 0 \ldots\left[d_{n+k-2}-1\right] 0\left[d_{n+k}-2\right] 0\left[d_{n+k+2}-1\right] 0\left[d_{n+k+4}-1\right] \ldots}^{-\left(d_{n}\right)} \\
n \in \mathbb{O}, k \in \mathbb{O} ; \\
\Delta_{\varepsilon_{1} \ldots \varepsilon_{n-1}\left[\varepsilon_{n}-1\right] 0\left[d_{n+2}-1\right] 0\left[d_{n+4}-1\right] 0 \ldots\left[d_{n+k-1}-1\right] 1\left[d_{n+k+1}-1\right] 0\left[d_{n+k+3}-1\right] \ldots}^{-\left(d_{n}\right)} \\
n \in \mathbb{E}, k \in \mathbb{O} ; \\
\Delta_{\varepsilon_{1} \ldots \varepsilon_{n-1}\left[\varepsilon_{n}-1\right] 0\left[d_{n+2}-1\right] 0 \ldots\left[d_{n+k-2}-1\right] 0\left[d_{n+k}-2\right] 0\left[d_{n+k+2}-1\right] 0\left[d_{n+k+4}-1\right] \ldots}^{-\left(d_{n}\right)} \\
n \in \mathbb{E}, k \in \mathbb{E}
\end{array}\right. \\
& x_{k}^{\prime \prime}=\left\{\begin{array}{l}
\Delta_{\varepsilon_{1} \ldots \varepsilon_{n-1}}^{-\left(d_{n}\right)}\left[\varepsilon_{n}-1\right] 0\left[d_{n+2}-1\right] 0 \ldots\left[d_{n+k-1}-1\right] 00\left[d_{n+k+2}-1\right] 0\left[d_{n+k+4}-1\right] \ldots \\
n \in \mathbb{O}, k \in \mathbb{O} ; \\
\Delta_{\varepsilon_{1} \ldots \varepsilon_{n-1}\left[\varepsilon_{n}-1\right] 0\left[d_{n+2}-1\right] 0 \ldots\left[d_{n+k}-1\right]\left[d_{n+k+1}-1\right] 0\left[d_{n+k+3}-1\right] 0\left[d_{n+k+5}-1\right] \ldots} n \in \mathbb{O}, k \in \mathbb{E} ; \\
\Delta_{\varepsilon_{1} \ldots \varepsilon_{n-1} \varepsilon_{n}\left[d_{n+1}-1\right] 0\left[d_{n+3}-1\right] \ldots 0\left[d_{n+k}-1\right]\left[d_{n+k+1}-1\right] 0\left[d_{n+k+3}-1\right] \ldots}^{-\left(d_{n}\right)} \\
n \in \mathbb{E}, k \in \mathbb{O} ; \\
\Delta_{\varepsilon_{1} \ldots \varepsilon_{n-1} \varepsilon_{n}\left[d_{n+1}-1\right] 0 \ldots\left[d_{n+k-1}-1\right] 00\left[d_{n+k+2}-1\right] 0\left[d_{n+k+4}-1\right] 0\left[d_{n+k+6}-1\right] \ldots}^{-\left(d_{n}\right)} \\
n \in \mathbb{E}, k \in \mathbb{E}
\end{array}\right.
\end{aligned}
$$

where $\mathbb{O}$ is a set of all odd positive integers and $\mathbb{E}$ is a set of all even positive integers.

That is,

$$
\begin{aligned}
& x_{k}^{\prime}=x_{0}^{(1)}+\frac{1}{d_{1} d_{2} \ldots d_{n+k}}, \\
& x_{k}^{\prime \prime}=x_{0}^{(2)}-\frac{1}{d_{1} d_{2} \ldots d_{n+k}},
\end{aligned}
$$

and $x_{k}^{\prime} \rightarrow x_{0}, x_{k}^{\prime \prime} \rightarrow x_{0}$ as $k \rightarrow \infty$.

Let $n$ be an odd number. Then

$$
\begin{aligned}
& y_{0}^{(1)}=g\left(x_{0}^{(1)}\right)=\Delta_{\varepsilon_{1}\left[d_{2}-1-\varepsilon_{2}\right] \varepsilon_{3}\left[d_{4}-1-\varepsilon_{4}\right] \varepsilon_{5} \ldots\left[d_{n-1}-1-\varepsilon_{n-1}\right] \varepsilon_{n}(0)}^{D}, \\
& y_{0}^{(2)}=g\left(x_{0}^{(2)}\right)=\Delta_{\varepsilon_{1}\left[d_{2}-1-\varepsilon_{2}\right] \varepsilon_{3}\left[d_{4}-1-\varepsilon_{4}\right] \ldots\left[d_{n-1}-1-\varepsilon_{n-1}\right]\left[\varepsilon_{n}-1\right]\left[d_{n+1}-1\right]\left[d_{n+2}-1\right]\left[d_{n+3}-1\right] \ldots}, \\
& y_{k}^{\prime}=g\left(x_{k}^{\prime}\right)=\Delta_{\varepsilon_{1}\left[d_{2}-1-\varepsilon_{2}\right] \varepsilon_{3}\left[d_{4}-1-\varepsilon_{4}\right] \ldots\left[d_{n-1}-1-\varepsilon_{n-1}\right] \varepsilon_{n}}^{D} \underbrace{0 \ldots 0}_{k-1} 1(0), \\
& y_{k}^{\prime \prime}=g\left(x_{k}^{\prime \prime}\right)=\Delta_{\varepsilon_{1}\left[d_{2}-1-\varepsilon_{2}\right] \varepsilon_{3}\left[d_{4}-1-\varepsilon_{4}\right] \ldots\left[d_{n-1}-1-\varepsilon_{n-1}\right]\left[\varepsilon_{n}-1\right]\left[d_{n+1}-1\right]\left[d_{n+2}-1\right] \ldots\left[d_{n+k}-1\right](0)}^{D}, \\
& \text { where } \tilde{F}(x)=F(g(x))=F \circ g \text {. }
\end{aligned}
$$


Hence,

$$
\begin{gathered}
\tilde{F}\left(x_{k}^{\prime}\right)=F\left(y_{k}^{\prime}\right)=\beta_{\varepsilon_{1}, 1}+\sum_{t=2}^{n-1}\left(\tilde{\beta}_{\varepsilon_{t}, t} \prod_{j=1}^{t-1} \tilde{p}_{\varepsilon_{j}, j}\right)+\beta_{\varepsilon_{n}, n} \prod_{j=1}^{n-1} \tilde{p}_{\varepsilon_{j}, j} \\
+\left(\sum_{l=n+1}^{n+k-1}\left(\beta_{0, l} \prod_{m=n+1}^{l-1} p_{0, m}\right)\right)\left(\prod_{j=1}^{n} \tilde{p}_{\varepsilon_{j}, j}\right)+\beta_{1, n+k}\left(\prod_{j=1}^{n} \tilde{p}_{\varepsilon_{j}, j}\right)\left(\prod_{m=n+1}^{n+k-1} p_{0, m}\right), \\
\tilde{F}\left(x_{0}^{(1)}\right)=F\left(y_{0}^{(1)}\right)=\beta_{\varepsilon_{1}, 1}+\sum_{t=2}^{n-1}\left(\tilde{\beta}_{\varepsilon_{t}, t} \prod_{j=1}^{t-1} \tilde{p}_{\varepsilon_{j}, j}\right)+\beta_{\varepsilon_{n}, n} \prod_{j=1}^{n-1} \tilde{p}_{\varepsilon_{j}, j} .
\end{gathered}
$$

Therefore,

$$
\tilde{F}\left(x_{k}^{\prime}\right)-\tilde{F}\left(x_{0}^{(1)}\right)=\beta_{1, n+k}\left(\prod_{j=1}^{n} \tilde{p}_{\varepsilon_{j}, j}\right)\left(\prod_{m=n+1}^{n+k-1} p_{0, m}\right)=\left(\prod_{j=1}^{n} \tilde{p}_{\varepsilon_{j}, j}\right)\left(\prod_{m=n+1}^{n+k} p_{0, m}\right) .
$$

In addition,

$$
\begin{gathered}
\tilde{F}\left(x_{0}^{(2)}\right)=F\left(y_{0}^{(2)}\right)=\beta_{\varepsilon_{1}, 1}+\sum_{t=2}^{n-1}\left(\tilde{\beta}_{\varepsilon_{t}, t} \prod_{j=1}^{t-1} \tilde{p}_{\varepsilon_{j}, j}\right)+\beta_{\varepsilon_{n}-1, n} \prod_{j=1}^{n-1} \tilde{p}_{\varepsilon_{j}, j} \\
+p_{\varepsilon_{n}-1, n}\left(\prod_{j=1}^{n-1} \tilde{p}_{\varepsilon_{j}, j}\right)\left(\sum_{l=n+1}^{\infty}\left[\beta_{d_{l}-1, l} \prod_{m=n+1}^{l-1} p_{d_{m}-1, m}\right]\right), \\
\tilde{F}\left(x_{k}^{\prime \prime}\right)=F\left(y_{k}^{\prime \prime}\right)=\beta_{\varepsilon_{1}, 1}+\sum_{t=2}^{n-1}\left(\tilde{\beta}_{\varepsilon_{t}, t}^{t-1} \prod_{j=1}^{t-1} \tilde{p}_{\varepsilon_{j}, j}\right)+\beta_{\varepsilon_{n}-1, n} \prod_{j=1}^{n-1} \tilde{p}_{\varepsilon_{j}, j} \\
+p_{\varepsilon_{n}-1, n}\left(\prod_{j=1}^{n-1} \tilde{p}_{\varepsilon_{j}, j}\right)\left(\sum_{l=n+1}^{n+k}\left[\beta_{d_{l}-1, l} \prod_{m=n+1}^{l-1} p_{d_{m}-1, m}\right]\right) .
\end{gathered}
$$

Hence,

$$
\tilde{F}\left(x_{0}^{(2)}\right)-\tilde{F}\left(x_{k}^{\prime \prime}\right)=p_{\varepsilon_{n}-1, n}\left(\prod_{j=1}^{n-1} \tilde{p}_{\varepsilon_{j}, j}\right)\left(\prod_{m=n+1}^{n+k} p_{d_{m}-1, m}\right) .
$$

Let $n$ be an even number. In this case,

$$
y_{0}^{(1)}=g\left(x_{0}^{(1)}\right)=\Delta_{\varepsilon_{1}\left[d_{2}-1-\varepsilon_{2}\right] \varepsilon_{3}\left[d_{4}-1-\varepsilon_{4}\right] \ldots \varepsilon_{n-1}\left[d_{n}-\varepsilon_{n}\right](0)}^{D},
$$




$$
\begin{gathered}
y_{0}^{(2)}=g\left(x_{0}^{(2)}\right)=\Delta_{\varepsilon_{1}\left[d_{2}-1-\varepsilon_{2}\right] \varepsilon_{3}\left[d_{4}-1-\varepsilon_{4}\right] \ldots \varepsilon_{n-1}\left[d_{n}-\varepsilon_{n}-1\right]\left[d_{n+1}-1\right]\left[d_{n+2}-1\right] \ldots}^{D}, \\
y_{k}^{\prime}=g\left(x_{k}^{\prime}\right)=\Delta_{\varepsilon_{1}\left[d_{2}-1-\varepsilon_{2}\right] \varepsilon_{3}\left[d_{4}-1-\varepsilon_{4}\right] \ldots \varepsilon_{n-1}\left[d_{n}-\varepsilon_{n}\right] \underbrace{D \ldots 0}_{k-1} 1(0)}^{D}, \\
y_{k}^{\prime \prime}=g\left(x_{k}^{\prime \prime}\right)=\Delta_{\varepsilon_{1}\left[d_{2}-1-\varepsilon_{2}\right] \varepsilon_{3}\left[d_{4}-1-\varepsilon_{4}\right] \ldots \varepsilon_{n-1}\left[d_{n}-1-\varepsilon_{n}\right]\left[d_{n+1}-1\right]\left[d_{n+2}-1\right] \ldots\left[d_{n+k}-1\right](0)}^{D} .
\end{gathered}
$$

Thus,

$$
\begin{gathered}
\tilde{F}\left(x_{k}^{\prime}\right)=F\left(y_{k}^{\prime}\right)=\beta_{\varepsilon_{1}, 1}+\sum_{t=2}^{n-1}\left(\tilde{\beta}_{\varepsilon_{t}, t} \prod_{j=1}^{t-1} \tilde{p}_{\varepsilon_{j}, j}\right)+\beta_{d_{n}-\varepsilon_{n}, n} \prod_{j=1}^{n-1} \tilde{p}_{\varepsilon_{j}, j} \\
\quad+\beta_{1, n+k}\left(\prod_{j=1}^{n-1} \tilde{p}_{\varepsilon_{j}, j}\right)\left(\prod_{m=n+1}^{n+k-1} p_{0, m}\right) p_{d_{n}-\varepsilon_{n}, n} \\
\tilde{F}\left(x_{0}^{(1)}\right)=F\left(y_{0}^{(1)}\right)=\beta_{\varepsilon_{1}, 1}+\sum_{t=2}^{n-1}\left(\tilde{\beta}_{\varepsilon_{t}, t}^{t-1} \tilde{p}_{j=1}^{t-1}\right)+\beta_{d_{n}-\varepsilon_{n}, n} \prod_{j=1}^{n-1} \tilde{p}_{\varepsilon_{j}, j} .
\end{gathered}
$$

Therefore,

$$
\begin{gathered}
\tilde{F}\left(x_{k}^{\prime}\right)-\tilde{F}\left(x_{0}^{(1)}\right)=\beta_{1, n+k}\left(\prod_{j=1}^{n-1} \tilde{p}_{\varepsilon_{j}, j}\right)\left(\prod_{m=n+1}^{n+k-1} p_{0, m}\right) p_{d_{n}-\varepsilon_{n}, n} \\
=\left(\prod_{j=1}^{n-1} \tilde{p}_{\varepsilon_{j}, j}\right)\left(\prod_{m=n+1}^{n+k} p_{0, m}\right) p_{d_{n}-\varepsilon_{n}, n} .
\end{gathered}
$$

In addition,

$$
\begin{aligned}
\tilde{F}\left(x_{0}^{(2)}\right)= & F\left(y_{0}^{(2)}\right)=\beta_{\varepsilon_{1}, 1}+\sum_{t=2}^{n-1}\left(\tilde{\beta}_{\varepsilon_{t}, t} \prod_{j=1}^{t-1} \tilde{p}_{\varepsilon_{j}, j}\right)+\beta_{d_{n}-1-\varepsilon_{n}, n} \prod_{j=1}^{n-1} \tilde{p}_{\varepsilon_{j}, j} \\
& +\left(\prod_{j=1}^{n} \tilde{p}_{\varepsilon_{j}, j}\right)\left(\sum_{l=n+1}^{\infty}\left[\beta_{d_{l}-1, l} \prod_{m=n+1}^{l-1} p_{d_{m}-1, m}\right]\right), \\
\tilde{F}\left(x_{k}^{\prime \prime}\right)= & F\left(y_{k}^{\prime \prime}\right)=\beta_{\varepsilon_{1}, 1}+\sum_{t=2}^{n-1}\left(\tilde{\beta}_{\varepsilon_{t}, t} \prod_{j=1}^{t-1} \tilde{p}_{\varepsilon_{j}, j}\right)+\beta_{d_{n}-1-\varepsilon_{n}, n} \prod_{j=1}^{n-1} \tilde{p}_{\varepsilon_{j}, j} \\
& +\left(\prod_{j=1}^{n} \tilde{p}_{\varepsilon_{j}, j}\right)\left(\sum_{l=n+1}^{n+k}\left[\beta_{d_{l}-1, l} \prod_{m=n+1}^{l-1} p_{d_{m}-1, m}\right]\right) .
\end{aligned}
$$


Hence,

$$
\tilde{F}\left(x_{0}^{(2)}\right)-\tilde{F}\left(x_{k}^{\prime \prime}\right)=p_{d_{n}-1-\varepsilon_{n}, n}\left(\prod_{j=1}^{n-1} \tilde{p}_{\varepsilon_{j}, j}\right)\left(\prod_{m=n+1}^{n+k} p_{d_{m}-1, m}\right) .
$$

Thus,

$B_{k}^{\prime}=\frac{\tilde{F}\left(x_{k}^{\prime}\right)-\tilde{F}\left(x_{0}\right)}{x_{k}^{\prime}-x_{0}}=\left\{\begin{array}{l}\left(d_{n} p_{\varepsilon_{n}, n}\right)\left(\prod_{j=1}^{n-1} d_{j} \tilde{p}_{\varepsilon_{j}, j}\right)\left(\prod_{m=n+1}^{n+k} d_{m} p_{0, m}\right), \\ n \text { is an odd; } \\ \left(d_{n} p_{d_{n}-\varepsilon_{n}, n}\right)\left(\prod_{j=1}^{n-1} d_{j} \tilde{p}_{\varepsilon_{j}, j}\right)\left(\prod_{m=n+1}^{n+k} d_{m} p_{0, m}\right), \\ n \text { is an even. }\end{array}\right.$

$B_{k}^{\prime \prime}=\frac{\tilde{F}\left(x_{0}\right)-\tilde{F}\left(x_{k}^{\prime \prime}\right)}{x_{0}-x_{k}^{\prime \prime}}=\left\{\begin{array}{l}\left(d_{n} p_{\varepsilon_{n}-1, n}\right)\left(\prod_{j=1}^{n-1} d_{j} \tilde{p}_{\varepsilon_{j}, j}\right)\left(\prod_{m=n+1}^{n+k} d_{m} p_{d_{m}-1, m}\right), \\ n \text { is an odd; } \\ \left(d_{n} p_{d_{n}-1-\varepsilon_{n}, n}\right)\left(\prod_{j=1}^{n-1} d_{j} \tilde{p}_{\varepsilon_{j}, j}\right)\left(\prod_{m=n+1}^{n+k} d_{m} p_{d_{m}-1, m}\right), \\ n \text { is an even. }\end{array}\right.$

Let us denote $b_{0, k}=\prod_{m=n+1}^{n+k} d_{m} p_{0, m}$ and $b_{d_{k}-1, k}=\prod_{m=n+1}^{n+k} d_{m} p_{d_{m}-1, m}$.

Since $\prod_{j=1}^{n-1} d_{j} \tilde{p}_{\varepsilon_{j}, j}=\mathrm{const}, p_{\varepsilon_{n}, n} p_{\varepsilon_{n}-1, n}<0, p_{d_{n}-\varepsilon_{n}, n} p_{d_{n}-1-\varepsilon_{n}, n}<0$ and the sequences $\left(b_{0, k}\right),\left(b_{d_{k}}-1, k\right)$ do not converge to 0 simultaneously (by the statement of the theorem), we obtain the following cases:

1. If the inequalities $d_{k} p_{0, k}>1$ and $d_{k} p_{d_{k}-1, k}>1$ hold for all $k \in \mathbb{N}$ except perhaps a finite set of numbers $k$, then one of the sequences $B_{k}^{\prime}, B_{k}^{\prime \prime}$ tends to $\infty$, and another sequence tends to $-\infty$;

2. If one of the products of $d_{k} p_{0, k}, d_{k} p_{d_{k}-1, k}$ is greater than 1 , and another is less than 1 for all $k \in \mathbb{N}$ except perhaps a finite set of numbers $k$, then one of the sequences $B_{k}^{\prime}, B_{k}^{\prime \prime}$ tends to $\pm \infty$, and another sequence tends to 0 ;

3. If one of the products of $d_{k} p_{0, k}, d_{k} p_{d_{k}-1, k}$ is greater than 1 , and another is equal to 1 for all $k \in \mathbb{N}$ except perhaps a finite set of numbers $k$, then one of the sequences $B_{k}^{\prime}, B_{k}^{\prime \prime}$ tends to $\pm \infty$, and another sequence is constant;

4. If one of the products of $d_{k} p_{0, k}, d_{k} p_{d_{k}-1, k}$ is less than 1 , and another is equal to 1 for all $k \in \mathbb{N}$ except perhaps a finite set of numbers $k$, then one of the sequences $B_{k}^{\prime}, B_{k}^{\prime \prime}$ tends to 0 , and another sequence is constant;

5. If the products of $d_{k} p_{0, k}, d_{k} p_{d_{k}-1, k}$ are equal to 1 for all $k \in \mathbb{N}$, then the sequences $B_{k}^{\prime}, B_{k}^{\prime \prime}$ are different constant sequences since the inequalities $p_{\varepsilon_{n}, n} \neq p_{\varepsilon_{n}-1, n}, p_{d_{n}-\varepsilon_{n}, n} \neq p_{d_{n}-1-\varepsilon_{n}, n}$ by the conditions $p_{\varepsilon_{k}, k} \in(-1 ; 1)$ and $\beta_{\varepsilon_{k}, k}>0$ for $\varepsilon_{k}>0$. 
Since $\lim _{k \rightarrow \infty} B_{k}^{\prime} \neq \lim _{k \rightarrow \infty} B_{k}^{\prime \prime}$ holds in all possible cases, it follows that the function $\tilde{F}$ is nowhere differentiable on $[0 ; 1]$.

\section{References}

[1] O.M. Baranovskyi, I.M. Pratsiovyta, and M.V. Pratsiovytyi, On One Function Related to First and Second Ostrogradsky Series. - Naukovyi Chasopys NPU im. M.P. Dragomanova. Ser. 1. Phizyko-matematychni Nauky [Trans. Natl. Pedagog. Mykhailo Dragomanov Univ. Ser. 1. Phys. Math.] 10 (2009), 40-49. (Ukrainian)

[2] M.V. Pratsiovytyi, Fractal Approach to Investigation of Singular Probability Distributions. Vydavnytstvo NPU im. M.P. Dragomanova [Dragomanov Nat. Pedagogical Univ. Publ.], Kyiv, 1998. (Ukrainian)

[3] M.V. Pratsiovytyi and A.V. Kalashnikov, On One Class of Continuous Functions with Complicated Local Structure, Most of which are Singular or Nondifferentiable. - Trudy Instituta Prikladnoi Matematiki i Mekhaniki NAN Ukrainy 23 (2011), 178-189. (Ukrainian)

[4] S.O. Serbenyuk, On One Nearly Everywhere Continuous and Nowhere Differentiable Function, that Defined by Automaton with Finite Memory. - Naukovyi Chasopys NPU im. M.P. Dragomanova. Ser. 1. Phizyko-matematychni Nauky [Trans. Natl. Pedagog. Mykhailo Dragomanov Univ. Ser. 1. Phys. Math.] 13(2) (2012), 166-182. (Ukrainian) Link: https://www.researchgate.net/publication/292970012

[5] S.O. Serbenyuk, Representation of Numbers by the Positive Cantor Series: Expansion for Rational Numbers. - Naukovyi Chasopys NPU im. M.P. Dragomanova. Ser. 1. Phizyko-matematychni Nauky [Trans. Natl. Pedagog. Mykhailo Dragomanov Univ. Ser. 1. Phys. Math.] 14 (2013), 253-267. (Ukrainian) Link: https://www.researchgate.net/publication/283909906

[6] S.O. Serbenyuk, On Some Sets of Real Numbers Such that Defined by Nega-sadic and Cantor Nega-s-adic Representations. - Naukovyi Chasopys NPU im. M. P. Dragomanova. Ser. 1. Phizyko-matematychni Nauky [Trans. Natl. Pedagog. Mykhailo Dragomanov Univ. Ser. 1. Phys. Math.] 15 (2013), 168-187. (Ukrainian) Link: https://www.researchgate.net/publication/292970280

[7] S.O. Serbenyuk, Defining by Functional Equations Systems of One Class a Functions, whose Arguments Defined by the Cantor Series. - International Mathematical Conference "Differential Equations, Computational Mathematics, Theory of Functions and Mathematical Methods of Mechanics" dedicated to 100th anniversary of G. M. Polozhy: Abstracts. - Kyiv, 2014. - P. 121. (Ukrainian) Link: https://www.researchgate.net/publication/301765329

[8] S.O. Serbenyuk, Functions, that Defined by Functional Equations Systems in Terms of Cantor Series Representation of Numbers. - Naukovi Zapysky NaUKMA 165 (2015), 34-40. (Ukrainian)

Link: https://www.researchgate.net/publication/292606546 
[9] A.F. Turbin and M.V. Pratsiovytyi, Fractal Sets, Functions, Probability Distributions. Naukova dumka, Kyiv, 1992. (Russian)

[10] G. Cantor, Ueber die Einfachen Zahlensysteme. - Z. Math. Phys. 14 (1869), 121-128. (German)

[11] A. Oppenheim, Criteria for Irrationality of Certain Classes of Numbers. - Amer. Math. Monthly. 61 (1954), No. 4, 235-241. 